\title{
Early medical abortion in Cairns, Queensland: July 2006 - April 2007
}

\author{
Caroline M de Costa, Darren B Russell, Naomi R de Costa, Michael Carrette and Heather M McNamee
}

M ifepristone (RU486) is not currently licensed for use in Australia. In December 2005, two of the authors ( $\mathrm{CM}$ de $C$ and $\mathrm{MC}$ ) made a joint application to the Therapeutic Goods Administration (TGA) for approval under the Authorised Prescriber legislation (Section 19[5] of the Therapeutic Goods Act 1989 [Cwlth]) to prescribe and supply mifepristone for the purpose of induced abortion, as we believed this option should be available for our patients. ${ }^{1}$ Here, we describe the process of obtaining Authorised Prescriber status and our early experience with mifepristone, and identify barriers to expanding access to mifepristone in Australia.

The main purpose of the Authorised Prescriber legislation is to allow Australian medical practitioners to use drugs that are available in other countries but not currently licensed in Australia. The drugs are for a patient or patients, exclusively within the practice of the applicant, whose clinical condition would benefit from the drug. Before making the actual application, a practitioner must obtain the approval of the ethics committee of either a hospital in which he or she works or a professional college - this approval is of the practitioner's suitability to prescribe the particular drug, rather than of the evidence for use of the drug itself.

Having obtained ethics committee approval, the practitioner, in making the application to the TGA, must include evidence for the safety and effectiveness of the drug, as well as detailed protocols for its use, copies of information to be supplied to patients, and details of consent procedures. TGA approval is conditional upon ongoing audit by the ethics committee of the use of the drug and regular reporting to the TGA. ${ }^{1}$ The TGA legislation limits all drugs administered by Authorised Prescribers to clinical indications that are "life-threatening or otherwise serious".

Approval of our suitability to administer the drug to appropriate patients resident in Cairns was obtained from the Cairns Base Hospital Ethics Committee. Cairns is a North Queensland city of 150000 people, with an annual birth rate of 3000. Our application to the TGA included an evidence-based list of medical conditions that would be a threat to the life, or a major threat to the health, of a pregnant woman continuing with the pregnancy. ${ }^{1}$ Our application was specifically for permission to use mifepristone together with misoprostol for induced abortion at up to 9 weeks' gestation, in accordance with the recommendations of the Royal College of Obstetricians and Gynaecologists (RCOG). ${ }^{2}$

At the time our application was made, under the Therapeutic Goods Amendment Act 1996 (Cwlth) (the "Harradine Amendment"), mifepristone could only be imported into or used in Australia with the personal permission of the federal Minister for Health. This unique piece of legislation had the effect of making the drug unavailable to Australian women from that date. However, in February 2006, a Private Members' Bill across party lines in the federal Parliament overturned the Harradine Amendment, and the decision about our application was thus made solely by the $\mathrm{TGA}^{3,4}$ In April 2006, we were granted permission to use the drug, and in June 2006, we were issued with a permit to import 40 tablets from the non-profit New Zealand company Istar Ltd, which

\section{ABSTRACT}

- Mifepristone (RU486), which is used for early medical abortion, can only be obtained in Australia under the Authorised Prescriber legislation (Section 19[5] of the Therapeutic Goods Act 1989 [Cwlth]); two of the authors have permission to obtain, prescribe and administer this drug in Cairns, Queensland.

- From July 2006 to April 2007, 10 women who fulfilled the Therapeutic Goods Administration (TGA) criteria of "lifethreatening or otherwise serious" indications underwent medical abortion with mifepristone/misoprostol, and 12 women conforming with abortion requirements of Queensland law, but not TGA legislation for mifepristone administration, had medical abortions with the less preferable methotrexate/misoprostol combination.

- Although it is now more than a year since the cross-party vote in federal Parliament in February 2006 confirmed wide support for the right of Australian women to a medical abortion, we believe we are at present the only medical practitioners in Australia with permission to use mifepristone.

- Obtaining Authorised Prescriber status from the TGA is of necessity a complex and protracted process, involving ethics committee approval and auditing, and regular reporting to the TGA.

- Because of the current restrictions, we believe that women seeking medical abortion in Australia face barriers not experienced by women in other comparable countries, and that drug manufacturing and distributing companies may be discouraged from seeking to market mifepristone in Australia.

MJA 2007; 187: 171-173

in turn had imported the drug from the French manufacturers, Exelgyn Laboratories.

These events generated considerable publicity, and in the past 12 months we have received more than 600 enquiries from women and doctors throughout Australia relating to the use of mifepristone for early abortion. These have been by telephone and email - our email addresses and telephone numbers being in the public domain - and have been in the approximate proportion of five requests from women seeking abortion to each request from a doctor either seeking information about the drug or wishing to refer a patient. We have, however, been constrained in our practice by the requirements of our TGA approval, and most of those making the requests were referred to abortion counselling services or providers of surgical abortion.

\section{Use of mifepristone/misoprostol in Cairns, July 2006 - April 2007}

In the first 10 months after receiving TGA permission, we performed 10 early medical abortions using the mifepristone/ 
misoprostol combination. The medical indications for these included: severe hypertension, severe pre-eclampsia/eclampsia in a previous pregnancy, thromboembolic disease requiring long-term anticoagulation, polycystic kidneys with recurrent urinary tract infections, previous ruptured uterus in pregnancy, severe depression requiring antidepressant therapy, recent major breast and pelvic floor surgery, and history of major fetal anomaly in a previous pregnancy. Most women had more than one indication.

All women had the alternatives to abortion explained in a consultation; in addition, the procedure was explained in detail, and informed consent obtained. All women had an intrauterine pregnancy of less than 63 days confirmed by ultrasound. The regimen recommended by the RCOG of $200 \mathrm{mg}$ mifepristone orally, followed 48 hours later by $800 \mu \mathrm{g}$ misoprostol intravaginally, was used in all cases. ${ }^{2}$

Nine women underwent the abortion process at home in the presence of a support person, while in close telephone contact with us and readily able to access emergency care if required. Analgesia and prophylactic antibiotics were prescribed. The abortions took place at home within 4 hours of the insertion of misoprostol, and there were no complications. The woman with a history of uterine rupture at 33 weeks' gestation in a previous pregnancy (after perforation of the uterus during an earlier surgical abortion) was admitted to hospital for the administration of misoprostol, and the abortion proceeded without complication.

All women were seen at a follow-up appointment, which included ultrasound scanning to confirm that the abortion was complete, plus advice on contraception and prevention of sexually transmitted infections.

\section{Use of methotrexate/misoprostol in Cairns, July 2006 - April 2007}

In the same time period, another 12 women requesting medical termination of pregnancy were seen at the Sexual Health Clinic in Cairns Base Hospital. The indications for abortion in these women, although conforming with the requirements of Queensland law, in our opinion fell short of those dictated by the TGA legislation for mifepristone administration. These women underwent appropriate consultations with two of us (DBR and $\mathrm{HMMcN}$ ) that were exactly the same as those provided to the women in the mifepristone group, and were administered methotrexate $50 \mathrm{mg}$ orally. Five to seven days later, $800 \mu \mathrm{g}$ misoprostol was inserted intravaginally. Again, the women underwent the abortion process at home with a support person present. One woman required surgical completion of an incomplete abortion; another woman bled vaginally for 7 days, but did not require transfusion or hospitalisation. Otherwise, the procedures were uncomplicated. The same follow-up measures were carried out as for the women given mifepristone/misoprostol.

\section{Expanding access to mifepristone in Australia}

In the period July 2006 - April 2007, there were approximately 2500 births in Cairns and 600 surgical abortions were performed. The number of medical abortions carried out under the Authorised Prescriber regulations therefore involved only a very small percentage of the pregnant women cared for in Cairns in this time. Our motive for reporting these cases is, firstly, to highlight the current clinical situation in Australia, which is little different from that preceding the overturning of the Harradine Amendment, and contrasts sharply with that in the United States, the United Kingdom, most of Europe, and elsewhere. Secondly, we wished to examine the barriers currently in existence that prevent or inhibit other practitioners from offering mifepristone to appropriate patients.

Medical abortion in early pregnancy using mifepristone/misoprostol, though widely available and largely non-controversial overseas, is, at the time of writing, still available in Australia only in Cairns and only within the very restricted framework of the Authorised Prescriber legislation. ${ }^{5-7}$ Medical abortion using methotrexate/misoprostol has also been widely and safely used overseas. ${ }^{5-8}$ This method is known to be used in Australia, but to date there have been no reports of this use in the medical literature. ${ }^{9}$ In December 2006, Marie Stopes International announced the results of a pilot program of methotrexate/misoprostol carried out at two clinics in New South Wales - 36 women had undergone a medical termination, with one woman subsequently requiring a surgical termination for a continuing pregnancy, but there were no other complications. ${ }^{10}$

The procedure for obtaining approval as an Authorised Prescriber of a drug from the TGA is of necessity a complex and protracted one. To date, through personal communication, we are aware of four applications from other practitioners for the use of mifepristone having been returned by the TGA with a request for further information, and, at the time of writing, no application has been approved for a pharmaceutical company to market the drug nationally. (The TGA has reportedly declined to state publicly whether any such applications have been received, as such material is commercial-in-confidence. ${ }^{11}$ )

We intend no criticism of the TGA in making these statements; we appreciate the thoroughly professional approach of TGA personnel in their evaluation of all drugs for use in Australia. In fact, the Authorised Prescriber legislation is an inappropriate route for large numbers of practitioners to access mifepristone; it can nevertheless be said that, in approving mifepristone for our use, the TGA has concluded from the evidence provided that the drug is sufficiently safe and effective to be available for at least some Australian women. However, the political debate surrounding the drug would appear so far to have had the effect of discouraging Australian drug manufacturing and distributing companies from seeking to market mifepristone in Australia.

A further potential disincentive lies in the fact that mifepristone, when used for induced abortion, is used in conjunction with misoprostol. Misoprostol is licensed for use in Australia, as elsewhere, only for the treatment of gastric ulceration, and therefore in all other situations is used "off-label" (ie, without specific TGA approval). ${ }^{12}$ Such use of drugs off-label is a common and accepted practice in Australian hospitals - misoprostol is widely used for the treatment of postpartum haemorrhage and, less commonly, for incomplete spontaneous miscarriage; and methotrexate, an anti-metabolite, is used for the treatment of unruptured ectopic pregnancy. ${ }^{13,14}$ However, the need to inform all patients of the off-label use of misoprostol, as our own protocols require, does make the process of consultation for medical abortion more prolonged, and could certainly complicate the application of a pharmaceutical company to market mifepristone in Australia.

\section{A call for proactive reform in access to abortion}

Our recent experience of providing early medical abortion in Cairns demonstrates vividly the conflicting situation facing women currently seeking medical abortion in Australia. Whereas the cross-party vote in the federal Parliament in February 2006, and 
the intense public discussion which surrounded it, confirmed wide support for the right of Australian women to medical abortion, a year later mifepristone use is restricted to a handful of women in Cairns, and our colleagues appear to be experiencing great difficulties obtaining approval to extend access to the drug. Methotrexate/misoprostol is being offered to some Australian women, although numbers are not known, and while this regimen is both legal and safe, overseas experience shows mifepristone/ misoprostol to be more effective and to have a shorter time frame for completion of the abortion process. ${ }^{15}$

Previous articles in the Journal have highlighted variations in abortion law between Australian states, calling for reform and a national approach to legislation and provision of abortion services. ${ }^{16,17}$ At present, because of variations in the accessibility of abortion among states and regions, many women are known to have travelled hundreds of kilometres to obtain abortions, often across state borders. ${ }^{18}$ If medical abortion were to become more easily available in only certain parts of the country, our experience suggests that such travel to obtain abortion would increase. National availability of mifepristone would potentially redress inequities of access to abortion services for Australian women, especially rural women and those from certain ethnic groups.

There is an urgent need for a more proactive approach from individual doctors, professional bodies and Health Departments, at both state and federal level, to make mifepristone widely used and largely uncontroversial, as it is in other countries, and accessible to every Australian woman faced with making the difficult decision about abortion for herself.

\section{Competing interests}

None identified.

\section{Author details}

Caroline M de Costa, FRANZCOG, FRCOG, MPH, Professor of Obstetrics and Gynaecology, Department of Obstetrics and Gynaecology ${ }^{1,2}$

Darren B Russell, FRACGP, DipVen, FAChSHM, Director, Sexual Health Clinic $^{1}$

Naomi R de Costa, LLB(Hons), GradDipLegalPrac, Solicitor ${ }^{3}$

Michael Carrette, MB BCh, FRANZCOG, Visiting Medical Officer ${ }^{1}$

Heather M McNamee, MB ChB, FRACGP, Visiting Medical Officer, Sexual Health Clinic ${ }^{1}$

1 Cairns Base Hospital, Cairns, QLD.

2 James Cook University School of Medicine, Cairns Campus, Cairns, QLD. 3 Edge Hill, Cairns, QLD.

Correspondence: caroline.decosta@jcu.edu.au

\section{References}

1 Therapeutic Goods Administration. Access to unapproved therapeutic goods - Authorised prescribers. http://www.tga.gov.au/docs/html/authpres.htm (accessed Jun 2007).

2 Royal College of Obstetricians and Gynaecologists. National Clinical Guidelines. The care of women requesting induced abortion. London: RCOG, 2004.

3 Bonner R. Debate on abortion pill in Australia becomes personal. The New York Times 2005; Feb 10: A3.

4 Zinn C. Health minister is stripped of his right to veto use of abortion pill. BMJ 2006; 332: 441.

5 American College of Obstetricians and Gynecologists. ACOG patient education. Induced abortion. http://www.acog.org/publications/patient_ education/bp043.cfm (accessed Jun 2007).

6 World Health Organization. Safe abortion: technical and policy guidance for health systems. Geneva: WHO, 2003. http://www.who.int/reproductive-health/publications/safe_abortion/index.html (accessed Jun 2007).

7 Creinin MD, Potter C, Holovanisin M, et al. Mifepristone and misoprostol and methotrexate/misoprostol in clinical practice for abortion. Am J Obstet Gynecol 2003; 188: 664-669.

8 Pymar HC, Creinin MD. Alternatives to mifepristone regimens for medical abortion. Am J Obstet Gynecol 2000; 183 (2 Suppl): S54-S64.

9 de Costa CM. Early medical abortion in Australia: more common than statistics suggest [letter]? Med J Aust 2006; 185: 341.

10 Marie Stopes International Australia. Medical abortion pilot program outcomes announced. Media release, 04/12/06. http://www.mariestopes.com.au/news/media_releases/medical_abortion_pilot_program_ outcomes (accessed Jul 2007).

11 Dobbin M. Abortion pill close. Freely available in months. Canberra Times 2007; Apr 15. http://canberra.yourguide.com.au/detail.asp?story_ id=575512 (accessed Jun 2007).

12 Queensland Health. Standard drug list (SDL) for Queensland hospitals (1 June 2007). http://www.foodsafetymatters.gov.au/qhcss/mapsu/documents/sdla.pdf (accessed Jun 2007).

13 Chong YS, Su LL, Arulkumaran S. Misoprostol: a quarter century of use, abuse, and creative misuse. Obstet Gynecol Surv 2004; 59: 128-140.

14 Gazarian M, Kelly M, McPhee JR, et al. Off-label use of medicines: consensus recommendations for evaluating appropriateness. Med J Aust 2006; 185: 544-548.

15 Say L, Kulier R, Gulmezoglu M, Campana A. Medical versus surgical methods for first trimester termination of pregnancy. Cochrane Database Syst Rev 2005; (1): CD003037.

16 de Crespigny LJ, Savulescu J. Abortion: time to clarify Australia's confusing laws. Med J Aust 2004; 181: 201-203.

17 de Costa CM. Medical abortion for Australian women: it's time. Med J Aust 2005; 183: 378-380.

18 Nickson C, Smith AM, Shelley J. Travel undertaken by women accessing private Victorian pregnancy termination services. Aust $N$ Z J Public Health 2006; 30: 329-333.

(Received 29 Jan 2007, accepted 6 May 2007) 